\title{
Comparison of high density and nitrogen seeded detachment using SOLPS-ITER simulations of the TCV tokamak
}

\author{
Smolders A. ${ }^{1,2}$, Wensing M. ${ }^{1}$, Carli S. ${ }^{2}$, De Oliveira H. ${ }^{1}$, \\ Dekeyser W. ${ }^{2}$, Duval, B.P. ${ }^{1}$ Février O. ${ }^{1}$, Gahle D. ${ }^{3}$, \\ Martinelli L. ${ }^{1}$, Reimerdes H. ${ }^{1}$, Theiler C. ${ }^{1}$, Verhaegh K. ${ }^{3}$ \\ and the TCV team ${ }^{4}$ \\ ${ }^{1}$ École Polytechnique Fédérale de Lausanne (EPFL), Swiss Plasma Center \\ (SPC), CH-1015 Lausanne, Switzerland, \\ ${ }^{2}$ KU Leuven, Department of Mechanical Engineering, Celestijnenlaan 300, 3001 \\ Leuven, Belgium \\ ${ }^{3}$ Culham Centre for Fusion Energy, Culham Science Centre, Abingdon, OX14 \\ 3DB, United Kingdom of Great Britain and Northern Ireland \\ ${ }^{4}$ See author list of S. Coda et al 2019 Nucl. Fusion 59112023 \\ E-mail: andreas.smolders@epfl.ch
}

12 September 2020

\begin{abstract}
First of a kind SOLPS-ITER simulations on TCV that include nitrogen have been performed to model recent nitrogen seeded detachment experiments. Based on spectroscopic measurements, a nitrogen recycling coefficient $R_{p}^{N} \approx 0.3-0.5$ on the graphite walls of TCV is estimated. The experimentally observed decrease of core nitrogen density with increasing plasma density is reproduced and linked to a reduction of the ionisation mean free path in the SOL. Although the influence of sputtered carbon impurities from TCV's graphite wall cannot be fully eliminated, seeding nitrogen increases control over the total impurity density. This facilitates disentangling the effect of impurities from that of high upstream density on the main characteristics of detachment, namely target power and ion current reductions and the development of a parallel pressure drop. Increasing the density and the seeding rate reduce the power on the divertor targets in a different way: with density, the ion current increases and the target temperature strongly decreases, whereas seeding impurities decreases the ion current and affects less strongly the temperature. The reduction in ion current when seeding nitrogen is due to a lower ionisation source, which is not related to power limitation nor an increased momentum loss, but to a decrease of the ionisation reaction rate. Impurity seeding leads to less volumetric momentum losses (and hence pressure drop) than density ramps, for the same level of energy flux reduction. Additionally, main chamber sputtering of carbon is identified as a possible explanation for the missing target ion current roll-over during density ramps in the simulations.
\end{abstract}




\section{Introduction}

In future high power magnetic fusion devices, like ITER and DEMO, the heat flux deposited on the wall will exceed material limits if not mitigated [1-3]. Strong dissipation of energy and momentum in the scrape-off layer (SOL) are therefore necessary and operation is envisioned in the detached regime [4-6]. Detachment is established by a number of processes that occur in low temperature divertors, including impurity radiation, plasma-neutral interactions and volumetric recombination [5]. Detachment studies on the Tokamak à Configuration Variable (TCV) [7] have focused so far on density ramps in L-mode plasmas in conventional and alternative divertor configurations [8-10]. TCV features a nearly complete graphite wall protection cover and carbon is always present in TCV plasmas with its source changing with plasma density. This leads to an increase of impurity radiation with density, up to $65 \%$ of the input power $[11,12]$. Recently, TCV has been upgraded to enhance reactor relevance [13]. In particular, heating power has been increased and divertor baffles have been installed to better separate the divertor from the main plasma and to reach regimes with higher divertor neutral pressures. In parallel, experiments employed nitrogen impurity seeding, as an alternative to density ramps, to achieve detachment [11] and to extend the studies to H-mode conditions [14]. Such active impurity seeding provides, in a carbon device, a more direct control on the impurity content than density ramps. It therefore helps disentangling the role of high plasma density from impurities for detachment and resembles more the situation in a metal device.

In accordance with most previous experiments, divertor detachment modelling on TCV has focused, to date, on density ramps [15-18]. The present work extends the modelling to impurity seeding. Simulations of nitrogen seeding were already performed for metal-walled tokamaks, including ASDEX-Upgrade [19-21] and ITER [21], where nitrogen was identified as a suitable divertor radiator for ITER.

The paper is structured as follows. In section 2 , the simulation set-up is presented together with the main diagnostics and experiments used in the comparison. In section 3 the recycling coefficient of $\mathrm{N}$ on TCV's graphite walls is estimated by comparison with spectroscopic data. The influence of density on nitrogen core penetration is discussed in section 4 . The main differences between nitrogen seeded and high density detachment are presented in section 5 .

\section{Simulation set-up}

The SOL and the outermost core region of TCV are simulated with the SOLPS-ITER code $[22,23]$ that employs the plasma fluid code B2.5 and the MonteCarlo neutrals code Eirene. It solves the multi-fluid balance equations for particles, parallel momentum and energy including simulated plasma interactions with kinetic neutrals. SOLPS-ITER is widely used in tokamak research and was the main tool to desgin ITER divertor [3].

In the present work, a lower single null configuration is modelled with a plasma current of $I_{p}=320$ $\mathrm{kA}$ and a nominal toroidal field of $1.43 \mathrm{~T}$ (fig. 1). This configuration is compatible with TCV's new divertor baffles but was performed before their installation [13]. The plasma density was ramped from a Greenwald fraction $f_{G} \approx 0.2$ to 0.65 through the discharge. In other discharges nitrogen was seeded while keeping density at a low $\left(f_{G} \approx 0.3\right)$ or high $\left(f_{G} \approx 0.5\right)$ Greenwald fraction.

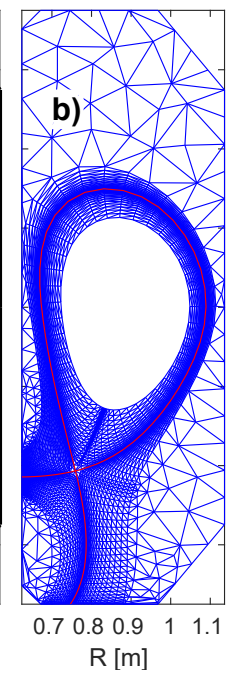

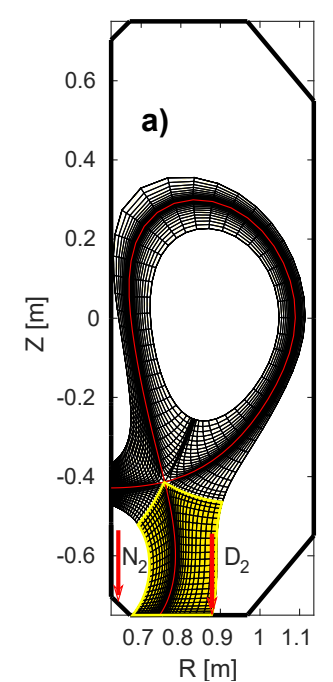

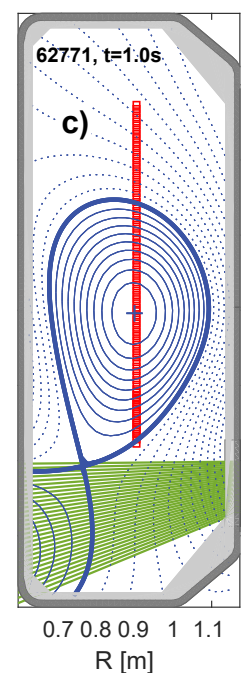

Figure 1: Set-up of the simulations and the experiments: a) B2.5 grid, gas valve locations and outer divertor region (yellow), b) Eirene grid and c) experimental set-up with Thomson chords $(\square)$ and the lines of sight of the divertor spectrometer system ( $\square)$.

The plasma species $\mathrm{D}^{+}, \mathrm{C}^{+}, \ldots \mathrm{C}^{6+}, \mathrm{N}^{+}, \ldots \mathrm{N}^{7+}$ and the neutral species $\mathrm{D}, \mathrm{D}_{2}, \mathrm{C}, \mathrm{N}$ are included in the simulation. A wide range of reactions is included by Eirene (tab. 1). Most notable omissions are molecular nitrogen and a possible formation of ammonia [24]. Nitrogen seeding is, thus, approximated by an influx of atomic $\mathrm{N}$ at the location of the gas valve in the private flux region (PFR) (fig. 1a).

Drift effects are not included and cross-field transport is approximated by empirically determined diffusivities $D_{\perp}=0.2 \mathrm{~m}^{2} / \mathrm{s}$ and $\chi_{\perp, i}=\chi_{\perp, e}=$ $1.0 \mathrm{~m}^{2} / \mathrm{s}$, as in $[15,16]$. This choice yields reasonable agreement with experimental Thomson scattering measurements of the upstream density and temperature profiles. 
Table 1: The interactions between neutrals and plasma particles included in Eirene together with their names in the code's databases.

\begin{tabular}{l|l} 
AMJUEL & Physical reaction \\
\hline H.4 H.10 2.1.5 & $\mathrm{D}+\mathrm{e} \rightarrow \mathrm{D}^{+}+2 \mathrm{e}$ \\
H.4 H.10 2.6A0 & $\mathrm{C}+\mathrm{e} \rightarrow \mathrm{C}^{+}+2 \mathrm{e}$ \\
H.4 H.10 2.7A0 & $\mathrm{N}+\mathrm{e} \rightarrow \mathrm{N}^{+}+2 \mathrm{e}$ \\
H.4 2.2.9 & $\mathrm{D}_{2}+\mathrm{e} \rightarrow \mathrm{D}_{2}^{+}+2 \mathrm{e}$ \\
H.4 2.2.5g & $\mathrm{D}_{2}+\mathrm{e} \rightarrow \mathrm{D}+\mathrm{D}+\mathrm{e}$ \\
H.4 2.2.10 & $\mathrm{D}_{2}+\mathrm{e} \rightarrow \mathrm{D}+\mathrm{D}^{+}+2 \mathrm{e}$ \\
H.0 H.1 H.3 0.3T & $\mathrm{D}_{2}+\mathrm{D}^{+} \rightarrow \mathrm{D}_{2}+\mathrm{D}^{+}$ \\
H.2 3.2.3 & $\mathrm{D}_{2}+\mathrm{D}^{+} \rightarrow \mathrm{D}_{2}^{+}+\mathrm{D}$ \\
H.4 2.2.12 & $\mathrm{D}_{2}^{+}+\mathrm{e} \rightarrow \mathrm{D}+\mathrm{D}^{+}+\mathrm{e}$ \\
H.4 2.2.11 & $\mathrm{D}_{2}^{+}+\mathrm{e} \rightarrow \mathrm{D}^{+}+\mathrm{D}^{+}+2 \mathrm{e}$ \\
H.4 H.8 2.2.14 & $\mathrm{D}_{2}^{+}+\mathrm{e} \rightarrow \mathrm{D}+\mathrm{D}^{+}$ \\
H.4 H.10 2.1.8 & $\mathrm{D}^{+}+\mathrm{e} \rightarrow \mathrm{D}(1 \mathrm{~s})$ \\
HYDHEL & $\mathrm{D}^{+}+2 \mathrm{e} \rightarrow \mathrm{D}(1 \mathrm{~s})+\mathrm{e}$ \\
\hline H.1 H.3 3.1.8 & $\mathrm{D}^{+}+\mathrm{D}(1 \mathrm{~s}) \rightarrow \mathrm{D}(1 \mathrm{~s})+\mathrm{D}^{+}$ \\
METHAN & \\
\hline H.1 H.3 3.2 & $\mathrm{D}^{+}+\mathrm{C} \rightarrow \mathrm{D}+\mathrm{C}^{+}$ \\
ADAS & \\
\hline H.4 acd96 & $\mathrm{C}^{+}+\mathrm{e} \rightarrow \mathrm{C}+h v$ \\
H.10prb96 & $\mathrm{N}^{+}+\mathrm{e} \rightarrow \mathrm{N}+h v$ \\
H.4 acd96 &
\end{tabular}

\section{$3 \quad$ Recycling coefficient estimate}

The plasma particle balance is determined by recycling coefficients and sputtering yields, in addition to pumps and gas injections. The TCV turbopumps have little influence on the particle balance during the 2 second discharge and only the machine walls are taken as particle sinks in the model. The recycling coefficient $R$, defined as the ratio of recycled neutral flux over the total wall flux (see section 4.6.3 in [4]), is set to 0.99 for deuterium, yielding reasonable agreement of the experimental and simulated gas puff flux for a given upstream density. The recycling coefficient of carbon is set to 0 and the chemical sputtering yield to $3.5 \%$ [25], in accordance with previous TCV modelling $[15,16]$.

Nitrogen ions and atoms exhibit a low level of recycling on both metal [26] and carbon walls [27-30], presumably due to their high chemical activity $[26$, 29]. Their recycling coefficient has, to the authors' knowledge, not been reported for carbon walls so this quantity needs to be estimated here. Before doing so, note that for a low nitrogen ion recycling coefficient $R_{p}^{N}$, the dominant neutral nitrogen source is the gas puff and not wall recycling (in steady state, the puffed fraction of the $\mathrm{N}$ source is $\left.1-R_{p}^{N}\right)$. Experimentally, the gas puff consists of $\mathrm{N}_{2}$ molecules, whereas the simulations only consider $\mathrm{N}$ atoms. To account for this, neutral $\mathrm{N}$ recycling is treated as $\mathrm{N}_{2}$ recycling. Contrarily to $\mathrm{N}$ ions, $\mathrm{N}_{2}$ is chemically stable and pulses without plasma indicate that $\mathrm{N}_{2}$ does not stick to the wall. Therefore, the neutral nitrogen recycling is set to $R_{n}^{N}=1$.

In order to estimate the nitrogen ion recycling coefficient, discharge 62893, a high density nitrogen seeding ramp, is compared with simulations where $R_{p}^{N}$ is varied from 0.1 to 0.8 . Upstream $n_{e}$ and $T_{e}$ profiles are matched with Thomson scattering measurements (fig. 2) and the input power is equal to the experimental Ohmic heating. This yields an overestimation of the target $J_{\text {sat }}$ by a factor 2-3 in the simulation, discussed further in section 5.2. A detailed comparison of $n_{e}$ and $T_{e}$ at the target is not possible due to known issues with the Langmuir probes in detached conditions [31].
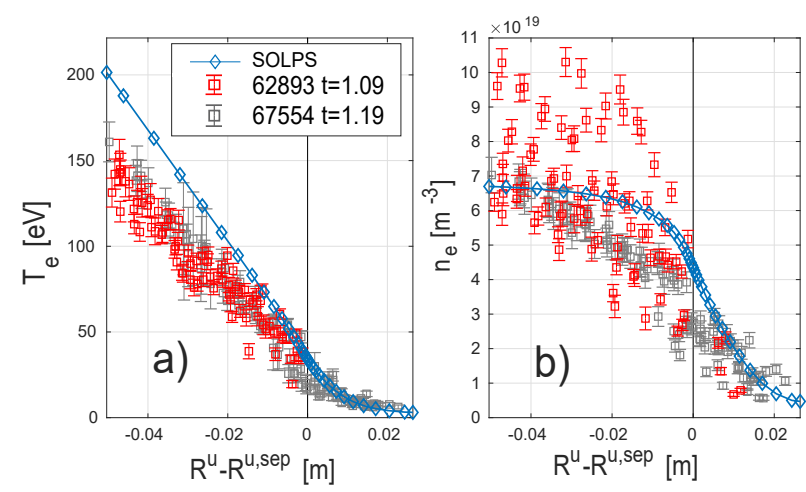

Figure 2: Comparison of upstream $T_{e}$ (a) and $n_{e}$ (b) profiles between the simulation and TCV discharges 62893 and 67544 . 67544 is a repeat of 62893 with slightly modified shape to improve the SOL profiles.

At constant $\mathrm{N}$ seeding rate, the divertor nitrogen ion content doubles when $R_{p}^{N}$ is increased from 0.1 to 0.5 , yielding an increase in divertor ionic nitrogen concentration from $1.5 \%$ to $2.8 \%$ for the highest seeding rate of $4 \times 10^{20} \mathrm{~N} / \mathrm{s}$. Note that these concentrations are significantly lower than for the low density simulation discussed in section 5 . While direct measurements of the nitrogen density are not available, the absolutely calibrated divertor spectrometer system (DSS) [32] can be used to probe consistency with simulations. For this purpose, synthetic diagnostic signals are calculated by line integrating the emissivity maps calculated by SOLPS for the 32 DSS view chords (fig. 1c) [33]. The local emissivity is calculated from the photon emission coefficients (PEC) based on [34]:

$\epsilon=\operatorname{PEC}^{\mathrm{exc}}\left(T_{e}, n_{e}\right) n_{e} n_{i}^{z+}+\mathrm{PEC}^{\mathrm{rec}}\left(T_{e}, n_{e}\right) n_{e} n_{i}^{(z+1)+}(1)$ with $\epsilon$ being the emissivity [photons $/ \mathrm{m}^{3} \mathrm{~s}$ ], $T_{e}$ the electron temperature $[\mathrm{eV}], n_{e}$ the electron density 
$\left[\mathrm{m}^{-3}\right]$ and $n_{i}^{z+}$ the ion density with ionisation state $z\left[\mathrm{~m}^{-3}\right]$. The emissivity of nitrogen lines is directly related to the nitrogen density, permitting an estimation of its recycling coefficient. Note that other empirical parameters, such as $R^{D}$, chemical sputtering yield or $D_{\perp}$ and $\chi_{\perp}$, also impact the nitrogen emissivity, but further analysis shows that their influence is either small or that changing these parameters deteriorates agreement with other experimental measurements, such as the $J_{\text {sat }}$ profile measured with Langmuir probes.

In fig. 3, DSS measurements of two NII and one NIII line are compared with synthetic measurements for different values of $R_{p}^{N}$ ranging from 0.1 to 0.8 for two seeding rates. Overall, the synthetic diagnostic signals are in good qualitative agreement with the experiments for both seeding rates except, close to the target, where the simulations underpredict the intensity for all values of $R_{p}^{N}$. This systematic underprediction may be related to an underestimation of the target $T_{e}$, underestimations of impurity density close to the target or inaccuracies in the PEC data at low temperature. The emissivity of each spectral line decreases sharply along the divertor leg towards the target. This corresponds to a characteristic electron temperature region, below which the emission coefficient decreases rapidly and the charge state corresponding to the emission is absent. The location of this drop in the simulations coincides with the measurements for multiple spectral lines, indicating that the temperature profile in the divertor up to this point is well described. In TCV, this sharp emission decrease is often used as a proxy for having obtained low divertor $T_{e}$ and, hence, conditions close to detachment $[9-11,14,35]$. Defining the radiation front location by a $50 \%$ decrease of the emissivity (as in $[9,10])$, the simulations indicate a front temperature of approximately 11-12 eV for NIII and CIII (not show) and $6.5 \mathrm{eV}$ for NII. Note that these temperatures are higher than estimates for a plasma simulated in coronal equilibrium due to the inclusion of impurity transport in these simulations.

While comparison of simulations with measurements in this TCV discharge indicates a recycling coefficient in the range $0.3-0.5$, repeated discharges indicate variations of $R_{p}^{N}$ with wall conditions. The range is in agreement with the partial recycling of $\mathrm{N}$ found on other machines [26-30]. In the following we take a fixed $R_{p}^{N}=0.3$, but the conclusions are not very sensitive to this value and also hold for $R_{p}^{N}=0.5$.

\section{Nitrogen core penetration}

In TCV, core radiation and $Z_{\text {eff }}$ increase strongly with nitrogen seeding at low, but not at high density [11].
This effect is reproduced in simulations for low and high density seeding ramps (fig. 4), similar to the low and high density seeding ramps on TCV (section 2). At low density, the $\mathrm{N}^{0}$ core ionisation and, hence, the nitrogen core ion density increase strongly with seeding (fig. 4a), while the increase is much weaker at higher density (fig. 4b). Consequently, the nitrogen core radiation and $Z_{\text {eff }}$ increase more strongly at low $n_{e}$ (fig. 4).

The larger increase in core ionisation at low density is explained by a reduction of the nitrogen ionisation mean free path $\lambda_{i o n, N}^{m f p}=v_{t, N} /\left(n_{e}\left\langle\sigma v_{e}\right\rangle_{i o n, N}\right)$ in the SOL with increasing plasma $n_{e}$ (fig. 5), with $v_{t, N}$ the thermal speed of atomic nitrogen. As the density increases, more neutrals ionise in the SOL and divertor before reaching the confined core plasma (fig. 5).

\section{Comparison of high density and nitrogen seeded detachment}

To investigate the differences in high upstream density and nitrogen seeded detachment, we start by comparing three cases: a low density attached case (LD), a high density case (HD) and a low density, nitrogen seeded case (NS) (tab. 2). All have an input power of $400 \mathrm{~kW}$ at the core boundary. The HD and NS cases are selected to have similar target power density profiles (fig. 6), making them equally favorable w.r.t the heat exhaust. In the NS case the fuelling is adjusted to match the upstream density profile of the LD case (fig. 7). The motivation behind this choice is that when starting from a reference scenario (here the LD case), both increasing upstream density or impurity content are possible options to mitigate target power loads. Ultimately, a good mix of both might be necessary, as seeding can cause undesired core radiation and dilution, while increasing upstream density typically degrades pedestal height and stability in the case of H-mode [36-38] and is limited by the Greenwald limit. Since the maximum target $T_{e}$ is below $5 \mathrm{eV}$ for both the HD and NS cases (fig. 9b), they are considered to be detached. They are not, however, equivalently detached, as only the target power is matched. Three characteristics of detachment are studied: a reduction of target power, a decrease of target ion current and the occurrence of a pressure drop parallel to the magnetic field.

\subsection{Target power}

Experimentally, the target power decreases both with increasing density and nitrogen seeding [11]. This is also found in the simulations, that can differentiate between the components of the target power and processes leading to its reduction. 


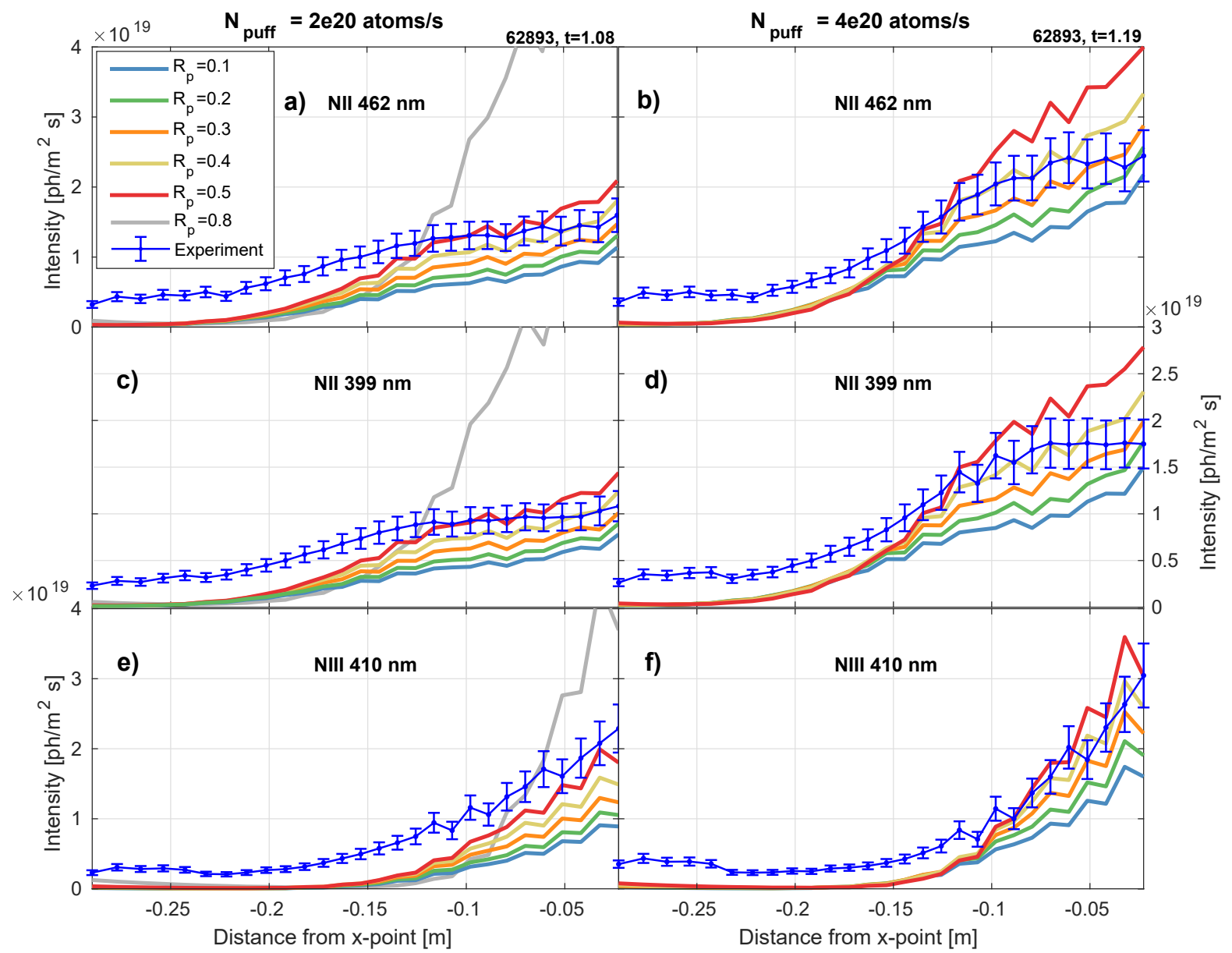

Figure 3: Comparison of the individual chord intensities of nitrogen emission lines as measured with the DSS and the synthetic DSS for simulations with different values of the nitrogen recycling coefficient $R_{p}^{N}$. Two different times of the experiment are shown at which the seeding rates are $1 \times 10^{20}$ and $2 \times 10^{20} \mathrm{molecules}_{2} / \mathrm{s}$. For the high seeding rate, simulations with $R_{p}^{N}>0.7$ resulted in a collapse of the upstream profile and are not shown.
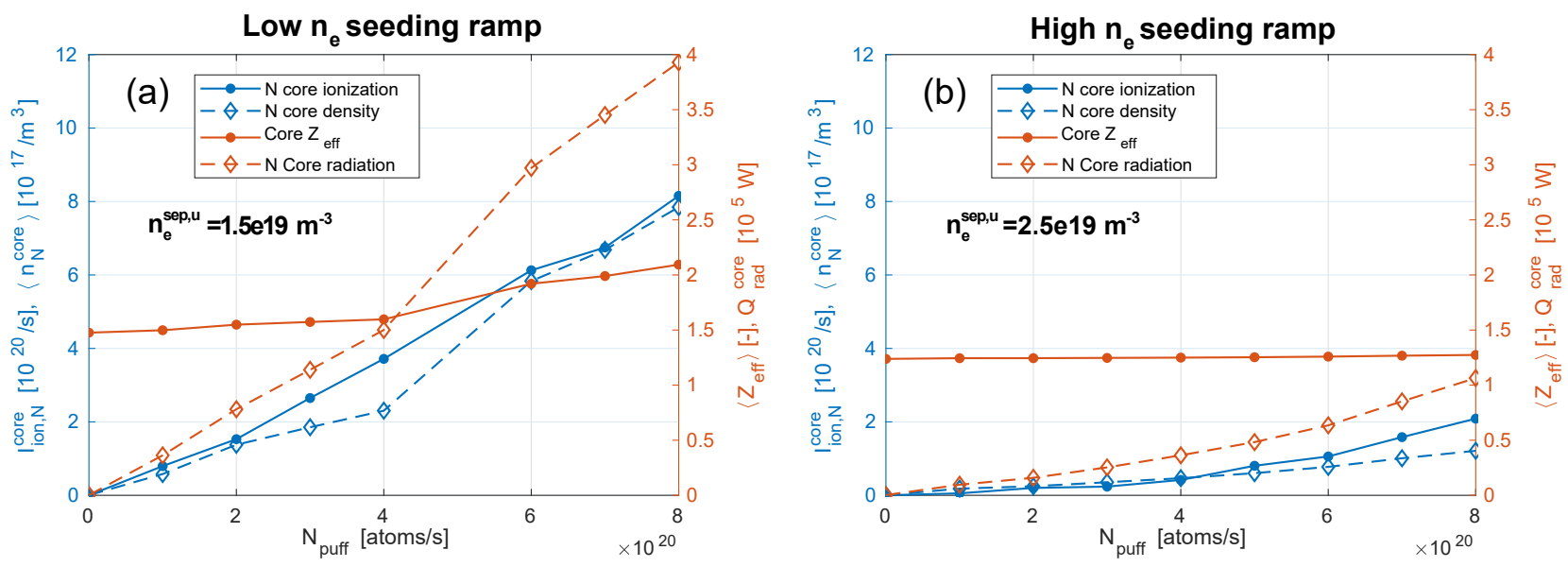

Figure 4: Neutral nitrogen core ionisation $I_{\text {ion }, N}^{\text {core }}$, average $\mathrm{N}$ core ion density $\left\langle n_{N}^{\text {core }}\right\rangle$, average core $\left\langle Z_{e f f}\right\rangle$ and $\mathrm{N}$ core radiation $Q_{\text {rad,N }}^{\text {core }}$ for (a) a low density and (b) a high density seeding ramp. All core quantities are evaluated over the simulated part of the core (fig. 1a). 


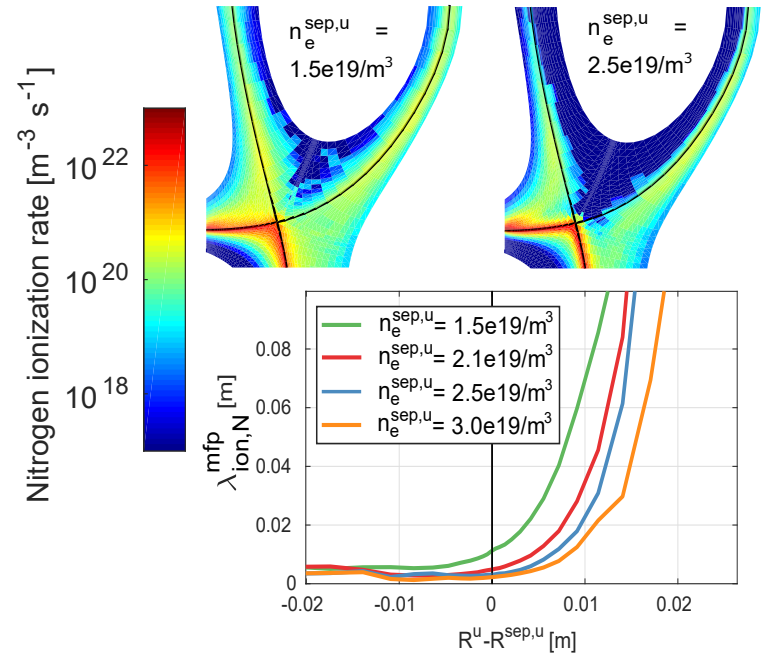

Figure 5: Neutral nitrogen ionisation rate and upstream mean free path $\left(\lambda_{i o n, N}^{m f p}\right)$ for simulations with increasing upstream separatrix density $n_{e}^{s e p, u}$. The seeding rate is constant at $4 \times 10^{20} \mathrm{~N} / \mathrm{s}$.

Table 2: Plasma parameters of the three cases studied in section 5 .

\begin{tabular}{l|ccc} 
Case & Low $n_{e}$ & High $n_{e}$ & N seeded \\
\hline$Q^{\text {ot }}[\mathrm{kW}]$ & 110.0 & 78.5 & 72.2 \\
$Q^{i t}[\mathrm{~kW}]$ & 152.5 & 96.9 & 99.6 \\
$n_{e}^{\text {sep }}, u\left[\mathrm{~m}^{-3}\right]$ & $1.51 \times 10^{19}$ & $2.75 \times 10^{19}$ & $1.57 \times 10^{19}$ \\
$T_{e}^{\text {sep }, u}[\mathrm{eV}]$ & 52.5 & 39.1 & 47.0 \\
$T_{e}^{\text {max }, o t}[\mathrm{eV}]$ & 9.7 & 1.0 & 4.6 \\
$T_{e}^{\text {max }, i t}[\mathrm{eV}]$ & 26.9 & 5.0 & 11.8 \\
$\frac{n_{0}^{\text {div }}\left[\mathrm{m}^{-3}\right]}{2.8 \times 10^{18}}$ & $1.6 \times 10^{19}$ & $3.3 \times 10^{18}$
\end{tabular}

5.1.1 Components of the target power The total target power density (fig. 6) is calculated as:

$q_{\perp}^{t}=\Gamma_{\perp}^{t} \gamma T^{t}+\Gamma_{\perp}^{t} \epsilon_{\mathrm{pot}}+q_{\perp}^{\text {rad }}+q_{\perp}^{\text {neut }}$

with $\Gamma_{\perp}^{t} \gamma T^{t}$ the kinetic plasma energy flux, $\Gamma_{\perp}^{t} \epsilon_{\text {pot }}$ the surface recombination contribution, $q_{\perp}^{\text {rad }}$ the energy flux linked to radiation, $q_{\perp}^{\text {neut }}$ the energy flux due to impinging neutrals and $q_{\perp}^{t}$ perpendicular to the target plate $\left[\mathrm{W} / \mathrm{m}^{2}\right] . \quad \Gamma_{\perp}^{t}$ denotes the perpendicular target particle flux $\left[1 / \mathrm{m}^{\frac{1}{2}} \mathrm{~s}\right], \gamma$ the sheath heat transmission coefficient, $T^{t}$ the target electron temperature and $\epsilon_{\mathrm{pot}}$ the potential energy from atomic recombination (13.6 $\mathrm{eV}$ for $\left.\mathrm{D}^{+}\right)$.

The simulations show that the power deposited by the plasma, consisting of the kinetic plasma energy flux $\left(\Gamma_{\perp}^{t} \gamma T^{t}\right)$ and the energy flux from surface recombination $\left(\Gamma_{\perp}^{t} \epsilon_{\mathrm{pot}}\right)$, dominates the total power density (fig. $8 \mathrm{a}$ and b). The power deposited by the plasma is approximately equal for both detached cases and largely reduced compared to the attached case. As the particle flux and hence the energy flux from surface

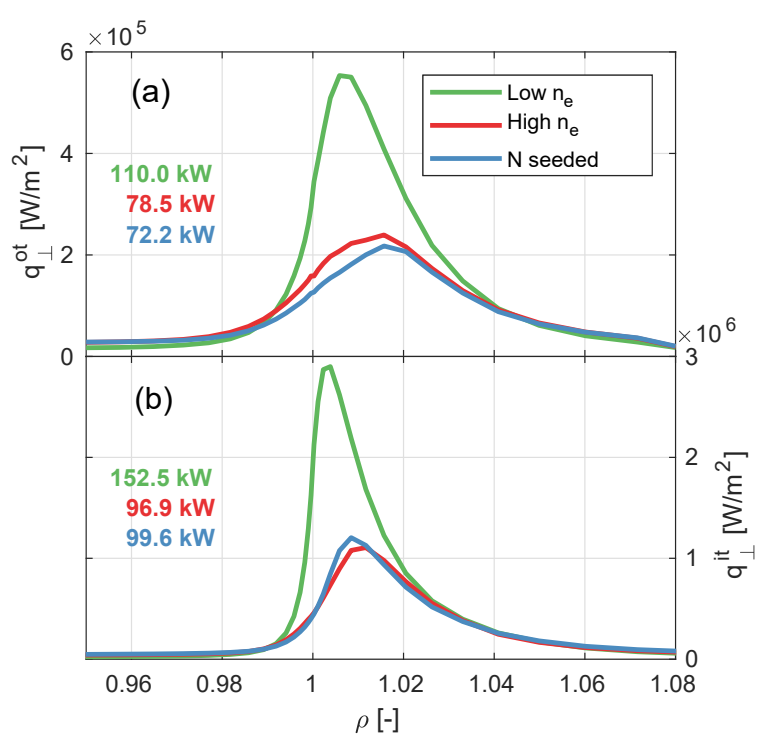

Figure 6: Outer (a) and inner (b) target power density profile for the three cases. The integrated target power is evaluated over the B2.5 plasma wetted area, see figure 1.

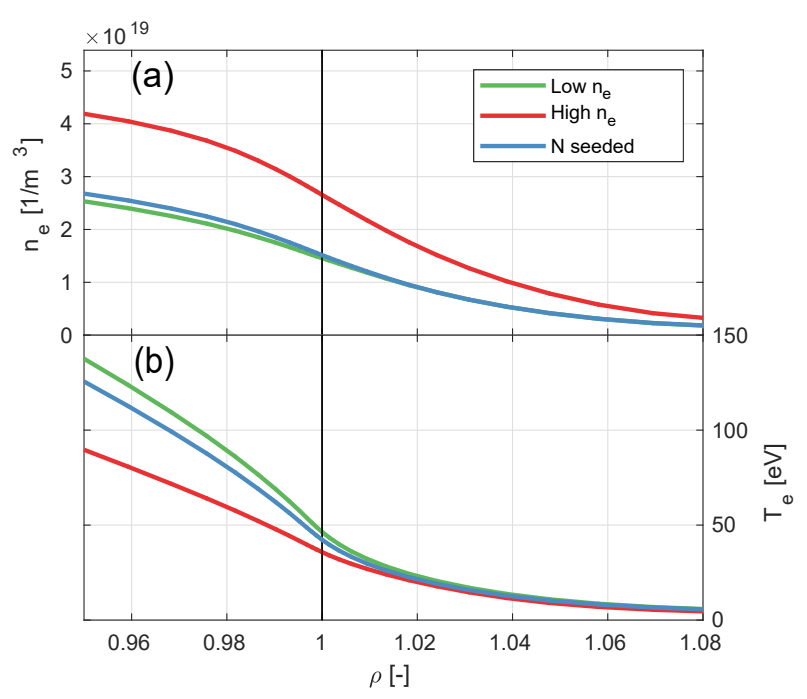

Figure 7: Upstream electron density (a) and temperature (b) profile for the three cases.

recombination is higher at high $n_{e}$ than with seeding (fig. 9a and 8b), the kinetic plasma energy flux and hence $T^{t}$ must be lower (fig. $9 \mathrm{~b}$ ).

The simulations reveal the importance of the contributions from radiation and neutrals to the target power density. For both detached cases, the $q_{\perp}^{\text {rad }}$ profile is similar. Although the integrated power on the target due to radiation is much higher for both detached cases, the peak energy flux is approximately equal in all three cases. This is explained by the radiation in attached 


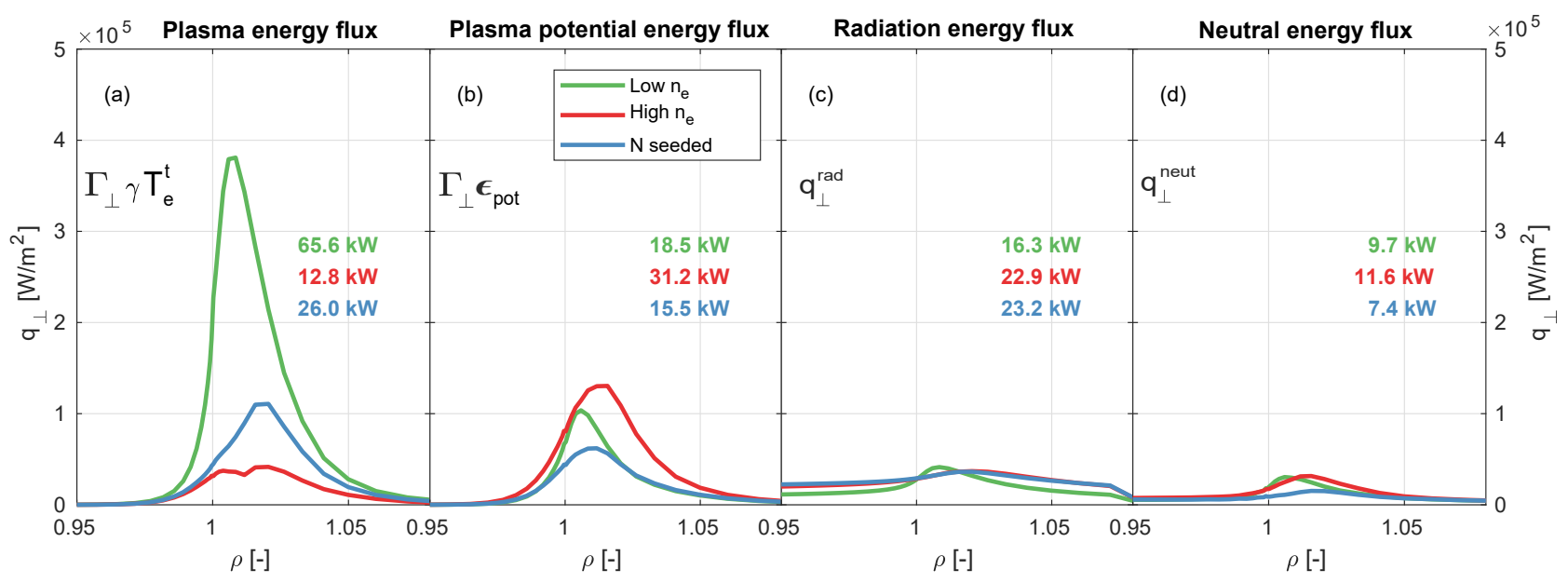

Figure 8: Radial profiles and integrated values of the components of the outer target power density. (a) Kinetic plasma energy flux, (b) plasma energy flux from surface recombination, (c) radiation energy flux and (d) energy flux carried by neutrals. The integrated power is evaluated over the B2.5 plasma wetted area, see figure 1 .

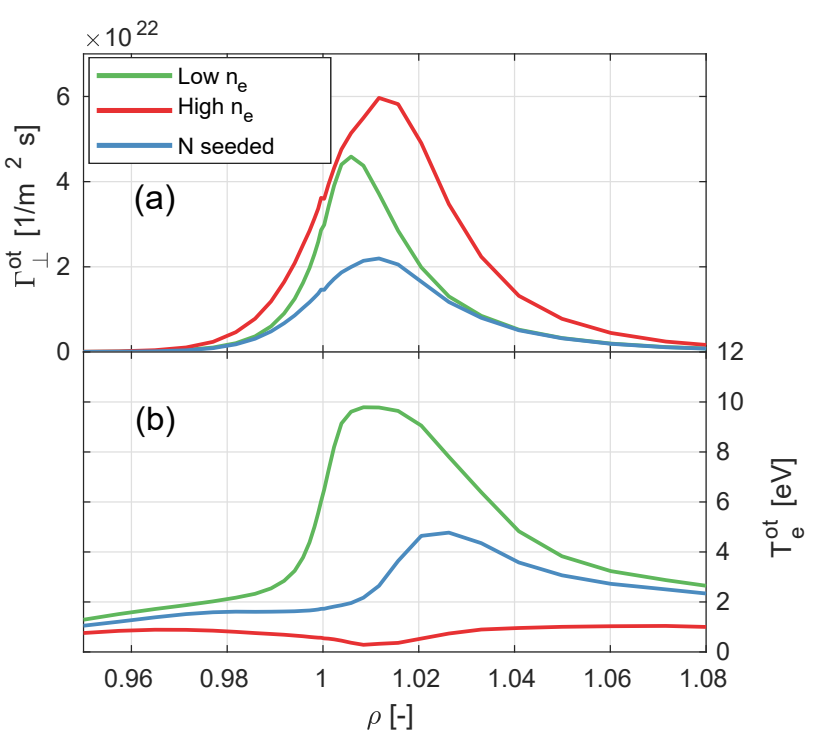

Figure 9: Outer target particle flux (a) and electron temperature (b) for the three cases.

conditions being very localised at the target, whereas the radiating region in the detached cases is located close to the X-point distributing the power over a larger surface. Neutrals carry less energy to the target than radiation, although the peak energy fluxes reach similar values. Overall, the power deposited on the targets by radiation and neutrals is not negligible. For the three cases studied, the combined contribution of radiation and neutrals to the peak power density is $12 \%$ for $\mathrm{LD}$, at $30 \%$ for $\mathrm{HD}$ and $23 \%$ for NS. This can partly explain regular mismatches between TCV heat flux estimates based on IR thermography and
Langmuir probes, since the former assumes a constant offset of power density instead of a profile to account for neutrals and radiation.

5.1.2 Volumetric power losses The reduction in target power with detachment results from an increase in radiation and plasma-neutral energy transfer. In the following, the contributions of both mechansims will be discussed. Although the peak $q_{\perp}$ can be further reduced by cross-field transport, this does not generate a volumetric energy sink.

Both detached cases show a strong increase in radiation compared to the attached case (fig. 10). Although the total radiative losses in the outer divertor are approximately equal for both cases, the contribution of each species is not. Without seeding, carbon radiation accounts for nearly $80 \%$ of the total radiation, consistent with experimental findings [12]. This radiation increases with upstream density, mainly due to the corresponding increase in divertor $n_{e}$, but also due to the higher carbon density from increased sputtering from increased particle fluxes on the walls (fig. 9a). For the NS case, nitrogen radiation dominates, accounting for $70 \%$ of the total radiation. This shows that in nitrogen seeded experiments, the main radiating species can indeed be controlled actively, which stresses the relevance of seeded TCV experiments for future, metal-walled reactors. Nitrogen injection is found to reduce the carbon radiation compared to the attached case. This is partly explained by a lower carbon density in the divertor, due to a lower particle flux (fig. 9a) and, hence, a lower the carbon sputtering rate. A reduction in divertor temperature displaces the radiating region 


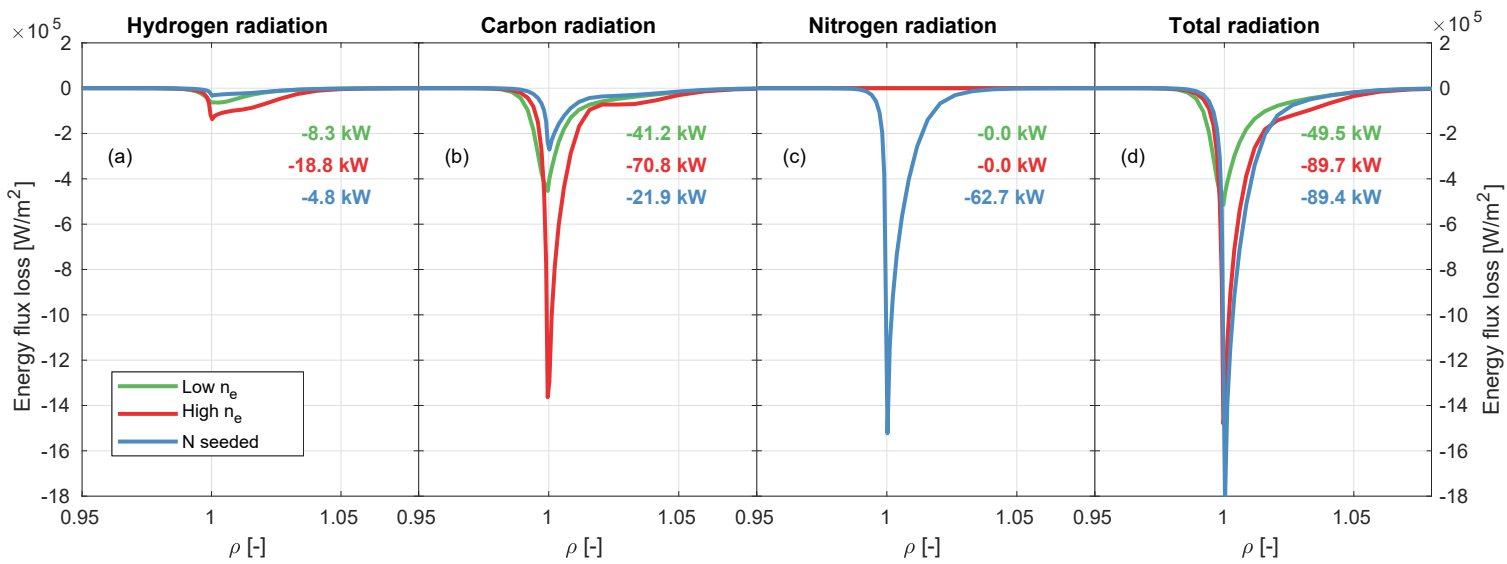

Figure 10: Radiation losses in the outer divertor: (a) hydrogen, (b) carbon, (c) nitrogen and (d) total radiation. The energy flux reduction is calculated by dividing the radiation energy losses along a certain flux tube from the $\mathrm{X}$-point to the target by the target area wetted by this flux tube, and can therefore be interpreted as the energy flux reduction by radiation in absence of radial transport in the divertor.

towards the main plasma, where the carbon density is found to be lower than at the target, further decreasing the radiated power.

The hydrogenic radiation is always considerably lower than that from impurities (fig. 10a). It increases significantly at high densities, continuing to account for $20 \%$ of the total radiated power, compared to only $5 \%$ for the NS case. Note that radiation from molecular bands together with radiation from excited atoms after plasma-molecule interactions are not included in the simulations. This value for the total hydrogenic radiation should thus only be considered as a lower bound.

The plasma neutral energy transfer can be divided into three parts: atomic charge exchange $(\mathrm{CX})$, energy transferred to molecules and plasma recombination (fig. 11). Details of their evaluation are discussed in Appendix A.

The power losses due to atomic CX in the outer divertor are similar for all cases (fig. 11a). In the common flux region (CFR), CX is mainly a loss mechanism as it involves cold recycling neutrals from the outer target. In the PFR, the simulations show that fast neutrals coming from the hot inner target (tab. 2) heat the plasma, most prominently at high density.

At high $n_{e}$, the molecular density close to the target increases strongly, consistent with expectations for low target $T_{e}$ [39]. Here, molecular losses dominate the neutral energy loss mechanisms, even surpassing hydrogenic radiation. At low $n_{e}$, with and without seeding, the target temperatures are higher and the molecular densities remain low, leading to a lower contribution from collisions with molecules to the power losses.

In conclusion, both an increase in density and impurity seeding lead to a strong reduction of the target power. According to the simulations, the dominating loss channel in TCV is impurity radiation: mainly from carbon for HD and mainly from nitrogen for NS. The neutral losses become important at low target $T_{e}$, mainly due to interactions with molecules, but remain significantly smaller than those from radiation. The volumetric power loss mechanisms at high density in TCV therefore fundamentally differ from metal-walled machines, where the impurity influx is independent of the particle flux.

\subsection{Ion current}

The experimental integrated target particle flux or ion current $I^{t}\left[\mathrm{~s}^{-1}\right]$ increases at the start of the density ramp and then rolls over at high $n_{e}[8,10$, 11]. In contrast, in nitrogen seeded discharges it decreases monotonically with increasing seeding [11]. The simulations reproduce the increase in $I^{t}$ with density (fig. 9a), but, as shown in a more extended density scan in this section, do not exhibit the rollover at high density. The simulated ion current is only found to stagnate and reduce marginally, and this at unrealistically high densities (not included here), similarly to previous SOLPS modelling of TCV [15]. The reduction of $I^{t}$ with seeding is, however, accurately reproduced, as apparent from fig. 9a and the more extended seeding scan discussed in the following.

To explain the different trend in $I^{t}$ between high density and nitrogen seeded detachment, we consider the equation for the ion current derived from the heat and particle balance in high recycling conditions (see 


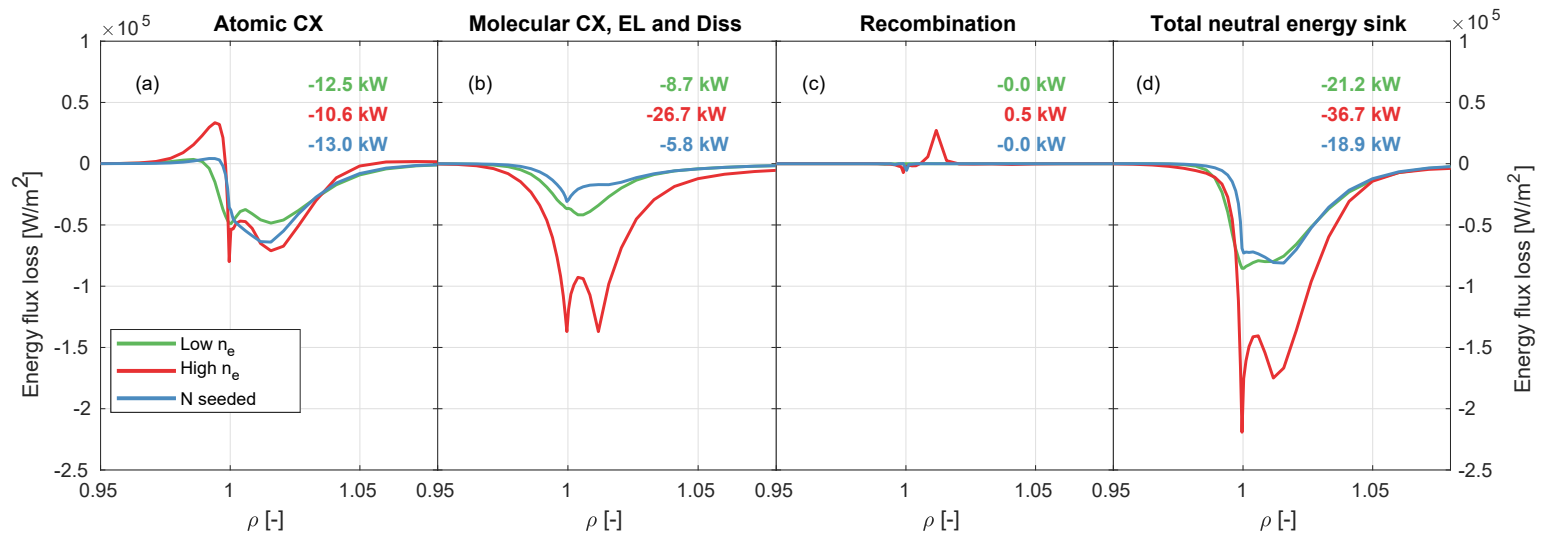

Figure 11: Plasma-neutral energy losses in the outer divertor: (a) the atomic charge exchange losses, (b) the molecular energy losses, consisting of molecular charge exchange, elastic collisions and molecular dissociation, (c) recombination losses and (d) the total neutral energy losses, i.e. the sum of (a), (b) and (c). The energy flux reduction is calculated the same way as in figure 10, meaning that a negative value represents volumetric power losses.

Appendix B and $[5,40])$ :

$I^{t}=\frac{Q_{\mathrm{IN}}^{\mathrm{div}}-Q_{\mathrm{imp}}-Q_{\mathrm{neut}}}{E_{\mathrm{ion}}+\gamma \overline{T^{t}}}-\frac{I_{\mathrm{rec}}}{1+\gamma \overline{T^{t}} / E_{\mathrm{ion}}}$

with $Q_{\mathrm{IN}}^{\text {div }}$ the incoming divertor power, $Q_{\text {imp }}$ the impurity radiation and $Q_{\text {neut }}$ the neutral energy losses, all in $[\mathrm{W}] . E_{\text {ion }}$ is the effective energy loss due to ionisation [12], accounting for both the hydrogenic radiation (fig. 10a) and the ionisation energy, $\bar{T}^{t}$ is a weighted average target electron temperature and $I_{\text {rec }}$ the recombination particle sink. Note that contrary to [5], the neutral losses are explicitly taken into account (fig. 11d). The individual terms of equation 3 are evaluated for simulated nitrogen seeding and density scans (fig. 12), where the quantities are integrated over the outer divertor and the outer part of the main SOL (fig. B1 in appendix).

In the nitrogen seeding scan, the seeding rate is increased from 0 to $8 \times 10^{20}$ atoms $/ \mathrm{s}$ for a constant $n_{e}^{\text {sep }, u}=1.5 \times 10^{19} / \mathrm{m}^{3}$, similar to the experimental low $n_{e}$ seeding ramp, reaching divertor nitrogen ion concentrations up to $15 \%$ (fig. 12a). For all seeding rates, recombination remains negligible, consistent with experimental observations [12]. Equation 3 can therefore be reduced to:

$$
\begin{aligned}
I^{t}=I_{\mathrm{ion}} & =\frac{Q_{\mathrm{IN}}^{\mathrm{div}}-Q_{\mathrm{imp}}-Q_{\mathrm{neut}}}{E_{\mathrm{ion}}+\gamma \overline{T^{t}}} \\
& =\int_{V} n_{e} n_{0}\langle\sigma v\rangle_{\mathrm{ion}}\left(n_{e}, T_{e}\right) d V
\end{aligned}
$$

where the ion current can also be written as the volume integrated local ionisation rate (eq. 5), with $n_{0}$ the atomic deuterium density, $\langle\sigma v\rangle_{\text {ion }}$ the ionisation rate coefficient and $V$ the volume (fig. B1 in appendix). The reduction in $I^{t}$ with seeding can be interpreted using both equation 4 and 5 . When seeding nitrogen, the radiated power increases (fig. 10d). Therefore, the target energy flux has to drop. This occurs, in part, through a reduction in the target $T_{e}$. Furthermore, at the start of the seeding ramp, the heat flux in the divertor is mainly electron conductive, so that $q_{\|} \underset{\sim}{\sim} \frac{\partial T_{e}}{\partial x}$ with $x$ the parallel direction, and the decrease in $q_{\|}$ flattens the parallel $T_{e}$ profile. Together with the lower target $T_{e}$, this results in a reduction in the divertor $T_{e}$ and, hence, a decrease of $\langle\sigma v\rangle_{\text {ion }}$ everywhere in the divertor. The simulations show that the electron density in the divertor remains approximately constant while the neutral density increases. The relative importance of the changes in $n_{e}, n_{0}$ and $\langle\sigma v\rangle_{\text {ion }}$ is illustrated in table 3 , showing that the increase in $n_{0}$ (second row) only partly compensates for the decrease in $\langle\sigma v\rangle_{\text {ion }}$ (third row). Therefore, the ionisation source and, hence, the target ion current decrease (eq. 5). Interpreted using equation 4, one concludes that, with the addition of nitrogen, the impurity radiation increases faster than the target temperature decreases, resulting in a decrease of $I^{t}$.

Table 3: Individual effect of changes in $n_{e}, n_{0}$ and $\langle\sigma v\rangle_{\text {ion }}$ on $I_{\text {ion }}$ (Eq. 5) in the density and seeding ramp. Cases $\mathrm{A}$ and $\mathrm{B}$ are indicated in figure 12. $\int n_{e}^{B} n_{0}^{A}\langle\sigma v\rangle_{\text {ion }}^{A} d V$ is the hypothetical ionization source if only $n_{e}$ would change going from A to B and $n_{0}$ and $\langle\sigma v\rangle_{\text {ion }}$ would remain constant.

\begin{tabular}{c|cc} 
& N seeding ramp & $n_{e}$ ramp \\
\hline $\int n_{e}^{B} n_{0}^{A}\langle\sigma v\rangle_{\text {ion }}^{A} d V / I_{\text {ion }}^{A}$ & 1.02 & 3.59 \\
$\int n_{e}^{A} n_{0}^{B}\langle\sigma v\rangle_{\text {ion }}^{A} d V / I_{\text {ion }}^{A}$ & 1.19 & 4.85 \\
$\int n_{e}^{A} n_{0}^{A}\langle\sigma v\rangle_{\text {ion }}^{B} d V / I_{\text {ion }}^{A}$ & 0.49 & 0.32
\end{tabular}


A reduction of $I^{t}$ is sometimes attributed to power limitation [12], a regime where the power required for ionisation $I_{\text {ion }} E_{\text {ion }}$ approaches the available $Q_{\mathrm{IN}}^{\text {div }}-$ $Q_{\text {imp }}-Q_{\text {neut }}$, constraining the ionisation source. From equation 4 , this happens when $\gamma \overline{T^{t}} \lesssim E_{\text {ion. }}$ This regime is not reached during the seeding ramp (fig. 12a), showing that, here, power limitation is not necessary for the target ion current to reduce. $I_{t}$ decreases as well in simulations with higher input power $(1.5 \mathrm{MW})$, in flux tubes with $T_{e}^{t}$ up to 15 $\mathrm{eV}$. Further simulations without carbon sputtering and with TCV's neutral gas baffles $[13,16]$ also exhibit similar behaviour, indicating the robustness of this result.

In another set of simulations, the midplane separatrix density is scanned from $1.5 \times 10^{19}$ to $4 \times$ $10^{19} / \mathrm{m}^{3}$ by increased $\mathrm{D}_{2}$ fuelling. At low density, the target $T_{e}$ is high, recombination is negligible and equation 4 is valid (fig. 12b). Increasing the gas fuelling initially leads to an enhanced ionisation rate (eq. 5) and hence $I^{t}$. According to equation 4, keeping all other quantities constant, the target temperature must drop. Additionally, increasing ionisation and, hence, plasma density increases the volumetric power losses (fig. 10 and 11), augmenting the temperature drop. In the simulations, the reduction of the ionisation rate coefficient $\langle\sigma v\rangle_{\text {ion }}$ due to this temperature decrease does not compensate for the increase in neutral and electron density (tab. 3), and the ionisation source, indeed, increases. In contrast to the nitrogen seeded case, equation 4 shows that, here, the target temperature decreases faster than the volumetric losses increase (fig. 12b), so $I^{t}$ increases.

At sufficiently high density, $\gamma \overline{T^{t}} \ll E_{\text {ion }}$ (fig. 12b) and equation 3 simplifies to [5]:

$I^{t}=\frac{Q_{\mathrm{IN}}^{\text {div }}-Q_{\mathrm{imp}}-Q_{\mathrm{neut}}}{E_{\mathrm{ion}}}-I_{\mathrm{rec}}=I_{\mathrm{ion}}^{\max }-I_{\mathrm{rec}}$.

Here, the target plasma power density $\left(q_{\perp}=\Gamma_{\perp}^{t}\left(\gamma T^{t}+\right.\right.$ $\left.\left.\epsilon_{\mathrm{pot}}\right)\right)$ is essentially proportional to the particle flux and, hence, the ionisation source. It cannot be reduced further by a drop in temperature. The ionisation source becomes constrained by the available power $\left(I_{\mathrm{ion}}^{\max }\right)$, previously referred to as power limitation.

Using equation 6 , we can probe the cause of the experimental roll-over by considering the differences between the experiments and SOLPS. In the following, different possible causes are discussed.

Volumetric recombination: According to eq. 6, volumetric recombination can result in a roll-over of $I^{t}$. However, volumetric electron-ion recombination is found to be small compared to ionisation in experiments [12] and simulations. Close to the target it may dominate ionisation, but its effect upon $I^{t}$ remains negligible. The $\mathrm{D}_{2}^{+}$branch of molecular activated recombination (MAR, [41]) is included in the simulations and found to dominate the total recombination in highly detached regimes, contrary to simulations of DIII-D like plasmas [42]. The lower divertor densities in these TCV plasmas may, however, explain why MAR is relatively more important. The importance of MAR in the experiments is subject of current research and the missing roll-over in our simulations could possibly be explained by an underestimation of the effect, since MAR is treated in a simplified way by SOLPS [42].

Increase in $E_{\text {ion }}$ : An increase of $E_{\text {ion }}$ was found to partially explain the experimental roll-over [12]. In the simulations, $E_{\text {ion }}$ ranges from 26 to $29 \mathrm{eV}$ during the density ramp (fig. 12b). This increase is small and therefore, alone, does not lead to a strong rollover. Spectroscopic estimates of $E_{\text {ion }}$ on TCV yield values up to $E_{\text {ion }} \sim 40 \mathrm{eV}$ [12]. This mismatch is mostly due to a change in volume over which $E_{\text {ion }}$ is calculated: in the experiment, only the outer divertor is considered, whereas the simulation also accounts for the main SOL contribution (fig. B1). Considering only the outer divertor, $E_{\text {ion }}$ increases up to $35 \mathrm{eV}$ in the simulation. Because of the similar variation in $E_{\text {ion }}$ in the simulations and the experiment, this effect is most likely not the cause of the missing roll-over in the simulations.

Increase of $Q_{\mathrm{imp}}+Q_{\text {neut }}$ : Experimentally it was found that the other main contributor to the rollover is an increase in power losses [12]. These losses are dominated by impurity radiation (fig. 10), that has been found to strongly reduce $I^{t}$, both on TCV with nitrogen seeding and other machines $[11,43]$. An underestimation of the impurity radiation in the simulations at high $n_{e}$ could explain why $I^{t}$ does not roll over (eq. 6). Carbon radiation, that dominates the losses in density ramps, depends on the carbon density, the electron density and the local radiation rate coefficient, which is a function of $n_{e}$ and $T_{e}$. Since $n_{e}$ and $T_{e}$ in the simulations match the Thomson scattering measurements, a difference in radiation is possibly due to the carbon density and/or its distribution within the plasma. Experimental core $\mathrm{C}^{6+}$ density measurements show an increasing trend when $I^{t}$ rolls over, while the simulations indicate that the core $\mathrm{C}$ content decreases with density. A possible explanation for this may be an increase in main chamber sputtering due to the observed broadening of the SOL at high density [11]. The energy of this sputtered carbon is higher than for target sputtering due to the higher upstream plasma temperature, increasing the ionisation mean free path and, hence, core penetration. This effect is not accounted for in SOLPS and could, thus, explain the discrepancy with the experiments [17]. To test this hypothesis, density ramp simulations were performed with an additional 

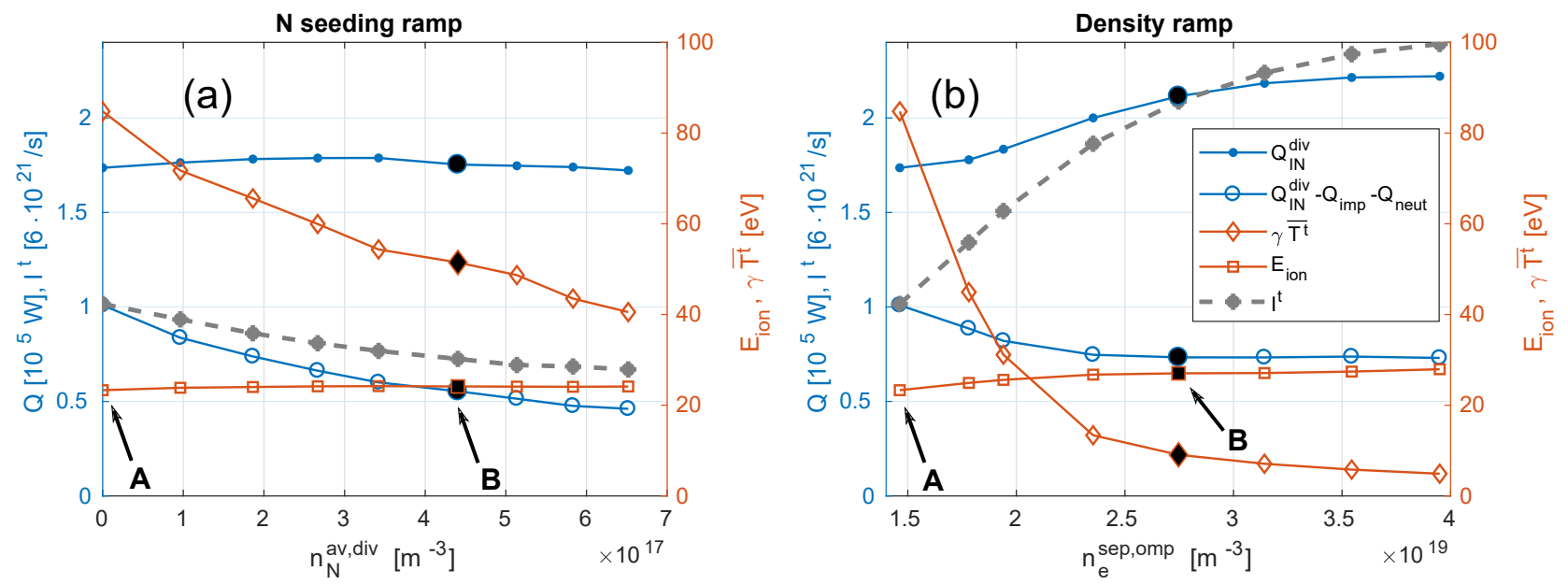

Figure 12: Components of equation 4 in the outer divertor (fig. B1) during (a) a seeding ramp as function of the average divertor nitrogen density and (b) a density ramp as function of the upstream separatrix density. The markers in black indicate the detached cases used for comparison in this section. The first simulation of both the seeding and density ramp is the same.

carbon source at the outer mid-plane, mimicking a main chamber sputtering source. Changing this source to enforce a constant carbon concentration of $\sim 5 \%$ in the outer SOL and divertor, indeed, results in a (weak) roll-over. Further support for this hypothesis stems from [44], where the ion current only rolled over in density ramps after the graphite coverage of the TCV walls was increased from $65 \%$ to $90 \%$, hence increasing main wall carbon sputtering.

In summary, both seeding nitrogen and increasing density reduce the plasma temperature and therefore $\langle\sigma v\rangle_{\text {ion. }}$. The simulations predict that, with seeding, this is not compensated by an increase in neutral and electron density, leading to a reduction of $I^{t}$. In contrast, at high density, it is overcompensated, leading to an increase of $I^{t}$.

Considering the different reasons for the absence of a roll-over at high $n_{e}$ in the simulations, the most likely explanation is an overestimation of the power available for ionisation, possibly due to the omission of main wall sputtering and, hence, a missing heat sink.

\subsection{Parallel pressure drop}

A parallel pressure drop between up- and downstream is observed both during density ramp and nitrogen seeding experiments, with, just before the disruption, a larger pressure drop in density ramps [11]. The simulations indicate that, for similar target power density, the pressure drop is indeed stronger with density than with seeding (fig. 13), but is less strong than in the experiments. This may be due to several reasons: (i) the cases presented here are less strongly detached, (ii) the experimental pressure drop is calculated over a larger part of the SOL and (iii) the pressure drop with seeding was only reported with seeding at high density in [11]. The processes causing the parallel pressure drop and the link with the target power and ion current are discussed in detail in the following.

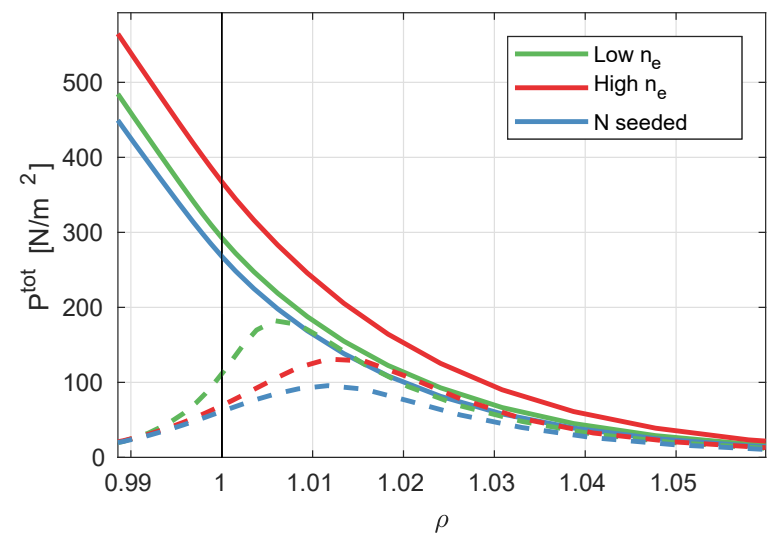

Figure 13: Upstream (solid) and downstream (dashed) total plasma pressure (see definition in app. Appendix C) for the three cases.

In analogy with [11], we define a pressure drop factor:

$f_{\text {pres }}^{\text {drop }}=\frac{\int p_{\text {tot }}^{t} \mathrm{~d} \rho}{\int p_{t o t}^{u} \mathrm{~d} \rho}=1-f_{\text {mom }}$

with $f_{\text {mom }}$ similar to the integral momentum loss factor defined in [45]. $f_{m o m}$ is dominated by neutral momentum losses. It has an additional geometric term, 
see Appendix C, causing deviations from pressure balance even in absence of volumetric momentum losses. $f_{\text {pres }}^{\text {drop }}$ is a measure of the integrated pressure loss, it has no contribution from radial momentum transport.

Atomic momentum losses increase strongly with $n_{e}$ and are considerably less affected when seeding nitrogen (fig. 14b). This is mainly due to the higher divertor neutral density in the high $n_{e}$ case compared to the other two cases (tab. 2). Also, molecular losses increase with density and are negligible in the low $n_{e}$ cases (fig. 14c). In this high density case, the atomic momentum losses are still twice as large as the molecular ones (see $f_{m o m}$ in figure 14), but at even higher density, molecular losses are found to dominate (not shown), consistent with previous SOLPS modelling on other machines [39, 46, 47].

Considering the parallel pressure drop with the results from previous sections yields two observations. First, a large reduction in target power can occur without a strong parallel pressure drop. Secondly, these simulations indicate that a strong parallel pressure drop is neither sufficient (high density), nor necessary (nitrogen seeded) for a reduction of $I^{t}$, the latter partly due to a drop in $p_{\text {tot }}^{u}$. The parallel pressure drop is found to correlate with low target $T_{e}$, consistent with expectations from [48], and not with a reduction of $I^{t}$. However, experimentally, it is observed that the reduction of $I^{t}$ occurs simultaneously with a drop in

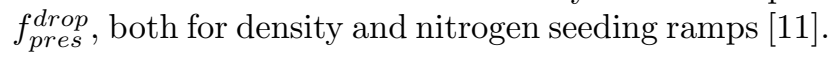

\section{Conclusion}

SOLPS-ITER simulations have been performed in order to study nitrogen seeded detachment on TCV. By matching experimental spectroscopic measurements of nitrogen line emissivities with synthetic diagnostic signals, the nitrogen recycling coefficient on the graphite walls of TCV is estimated to be $R_{p}^{N} \approx$ $0.3-0.5$. Additionally, it was determined that the experimentally observed decrease in impurity core penetration with increasing density is due to screening of the core by ionisation of impurity neutrals in the high density SOL. Seeding nitrogen allows to vary the impurity content in the SOL, unlike for high density detachment, allowing a separation of the effects of high density and high impurity content. Detached simulations with similar target power, achieved with either high density or nitrogen seeding, are compared. Firstly, with seeding, the target $T_{e}$ is higher and the target energy flux reduction is mainly due to a reduction of the particle flux, whereas for density ramps, it is mainly due to a decrease in the target $T_{e}$. Secondly, a reduction of the target ion current with nitrogen seeding is found, in accordance with the experiments. This is found not to be related to power limitation, but to a reduction in $\langle\sigma v\rangle_{\text {ion }}$. For the density ramp, the power limitation regime is quickly attained, but $I^{t}$ does not roll over, in contrast with experiments. A possible cause of this difference is a strong additional carbon source from main wall sputtering in the experiment. Finally, for equal target power, a pressure drop along the SOL is found to be stronger at high density than with seeding. This is due to the lower target $T_{e}$ at high density leading to a higher neutral density and, hence, higher momentum losses. A parallel pressure drop is, therefore, not necessarily related to an ion current reduction but mainly to the divertor temperature.

\section{Acknowledgments}

The authors would like thank David Moulton for the SOLPS balance routines. This work has been carried out within the framework of the EUROfusion Consortium and has received funding from the Euratom research and training programme 2014-2018 and 2019-2020 under grant agreement No 633053. The views and opinions expressed herein do not necessarily reflect those of the European Commission. This work was supported in part by the Swiss National Science Foundation. The computational resources and services used in this work were provided by the VSC (Flemish Supercomputer Center), funded by the Research Foundation Flanders (FWO) and the Flemish Government - department EWI.

\section{Appendix A Calculation of the neutral energy losses}

SOLPS solves the internal energy equation, so the sources in the fluid equations are converted to total energy sources to estimate the neutral energy losses:

$$
S^{E}=S_{i}^{H}+S_{e}^{H}+\sum_{a}\left(u_{a} \cdot S_{a}^{m o m}-\frac{1}{2} m_{a} u_{a}^{2} S_{a}^{\text {particle }}\right)
$$

with $S^{E}$ the total energy source, $S_{i}^{H}$ and $S_{e}^{H}$ the ion and electron the heat sources, $a$ the species index, $u_{a}$ the parallel velocity, $S_{a}^{\text {mom }}$ the parallel momentum source, $m_{a}$ the atomic mass of species $a$ and $S_{a}^{p a r t i c l e}$ the particle source. The EIRENE sources from atoms, molecules, test ions $\left(\mathrm{D}_{2}^{+}\right)$and recombination can be distinguished. The total molecular losses are calculated by summing up the molecular and test ion sources.

Section 5.1 identifies the loss channels which result in a reduction of the power on the targets. The potential energy needed for the ionisation of atoms is not a volumetric loss channel, it represents only the conversion of internal energy (i.e. heat) into potential 


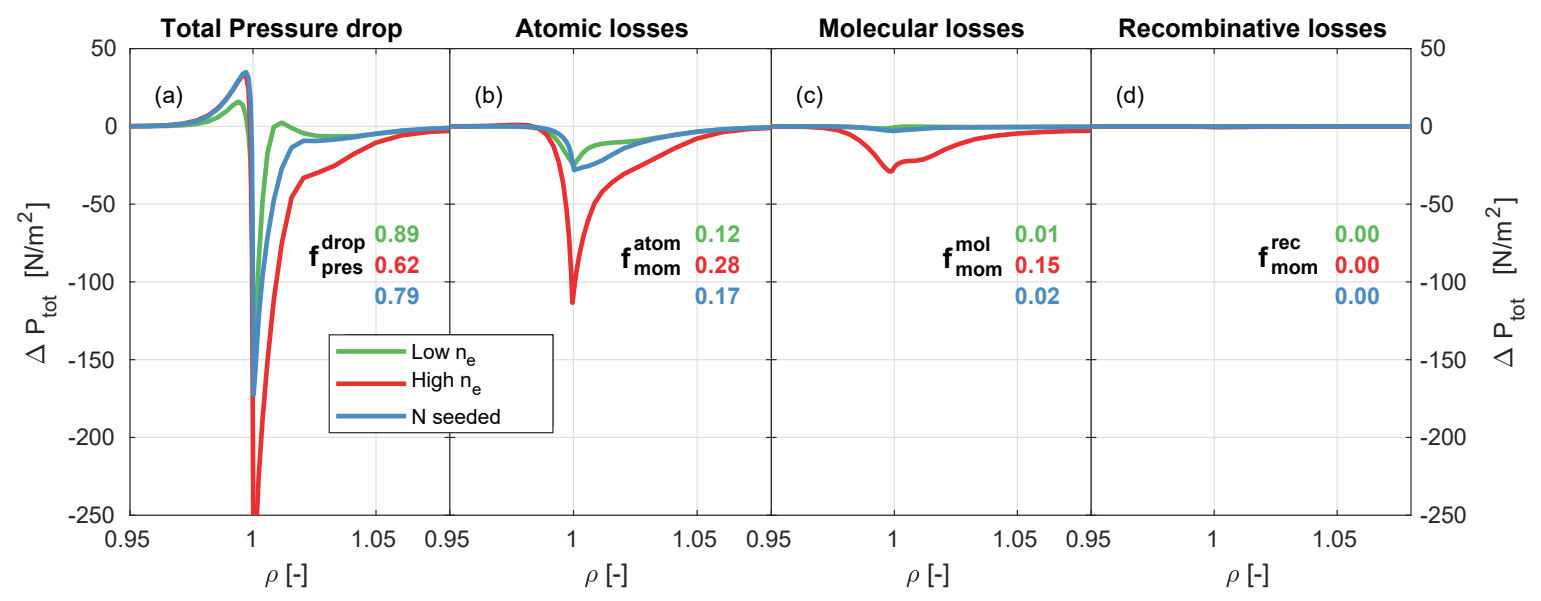

Figure 14: Parallel pressure drop between the entrance of the outer divertor and the outer target: (a) the radial profile of the total pressure drop, together with the factor $f_{\text {pres }}^{\text {drop }}$ (indicated with coloured numbers), (b) atomic, (c) molecular and (d) recombinative contribution to the parallel pressure drop, with their contributions to $f_{\text {mom }}$ (indicated with coloured numbers).

energy and, since the ions and electrons are trapped in the plasma, this energy is still deposited on the targets. Therefore, it is removed from $S^{E}$. Additionally, the energy radiated by neutrals was already considered in the radiation losses and is therefore also subtracted.

The potential energy for the dissociation of hydrogen molecules is, contrary to the ionisation energy, not always deposited on the targets. The two dissociated D atoms are not trapped in the plasma and, since the ionisation mean free path is relatively large in these TCV plasmas, these atoms can escape the plasma and deposit the dissociation energy on the main wall. This can be an important loss channel because the dissociation of $\mathrm{D}_{2}$ by electron impact is the dominating $\mathrm{D}_{2}$ reaction below $T_{e}=10 \mathrm{eV}$ [49]. However, if the atoms are subsequently ionised after dissociation, the dissociation energy will be deposited on the targets. It is chosen to take the molecular dissociation energy as volumetric losses, although this is an overestimation of the real target power reduction.

\section{Appendix B Derivation of the equation for the ion current}

Following [5], equation 3 is derived from the particle and power balance over a part of the plasma (fig. B1):

$I^{t}=I_{\mathrm{IN}}^{\text {div }}+I_{\text {ion }}-I_{\text {rec }}$

$Q^{t}=Q_{\mathrm{IN}}^{\mathrm{div}}-Q_{\mathrm{imp}}-Q_{\text {neut }}-Q_{\mathrm{H}}$

with $I^{t}$ the target ion current, $I_{\mathrm{IN}}$ div the incoming ions, $I_{\text {ion }}$ the ionisation source and $I_{\text {rec }}$ the recombination sink. $Q^{t}$ is the target kinetic power and can be rewritten as $\gamma \overline{T^{t}} I^{t}$ with $\gamma$ the sheath heat transmission coefficient and $\overline{T^{t}}$ the weighted average target electron temperature. In figure $12, \gamma \overline{T^{t}}$ is calculated as $Q^{t} / I^{t}$, therefore describing an energy flux weighted average $T^{t}$. $Q_{\mathrm{IN}}^{\mathrm{div}}$ is the incoming power, $Q_{\mathrm{imp}}$ is the impurity radiation, $Q_{\text {neut }}$ is the energy transferred to neutrals and $Q_{\mathrm{H}}$ is the energy lost from the plasma due to the ionisation of hydrogen, including the potential energy for ionisation and the hydrogenic radiation. $Q_{\mathrm{H}}$ can be rewritten as $I_{\text {ion }} E_{\text {ion }}$, with $E_{\text {ion }}$ the average energy used per ionisation event, which is dependent on temperature. Note that in [5], the neutral energy losses are taken proportional to the ionisation rate, i.e $Q_{\text {neut }}=I_{\text {ion }} E_{\mathrm{CX}}$, leading to an effective cost of ionisation $E_{\text {eff }}=E_{\text {ion }}+E_{\mathrm{CX}}$, whereas here, the losses are explicitly considered. Assuming that $I_{\mathrm{IN}}^{\mathrm{div}}$ is much smaller than $I_{\text {ion }}-I_{\text {rec }}$ because of the high recycling conditions, combining equation B.1 and B.2 yields:

$$
I^{t}=\frac{Q_{\mathrm{IN}}^{\mathrm{div}}-Q_{\mathrm{imp}}-Q_{\mathrm{neut}}}{E_{\mathrm{ion}}+\gamma \overline{T^{t}}}-\frac{I_{\mathrm{rec}}}{1+\gamma \overline{T^{t}} / E_{\mathrm{ion}}} .
$$

The values shown in figure 12 are integrated over the outer divertor and the outer SOL (fig. B1). For all other figures presented above, only the outer divertor is taken, i.e. from the $\mathrm{X}$-point to the target. As the main SOL contributes to a large fraction of the ionisation, not taking into account this part would violate the assumption that $I_{\text {ion }}-I_{\text {rec }} \gg I_{\text {IN }}^{\text {div }}$. Taking this volume, $I_{\mathrm{IN}}^{\mathrm{div}}$ is approximately one order of magnitude below $I^{t}$. 


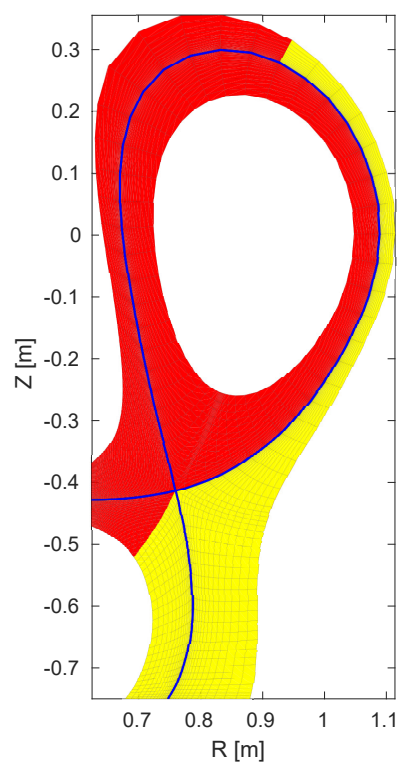

Figure B1: The volume indicated in yellow is taken for the integration of the quantities in equation 3 and B.3.

\section{Appendix C Derivation of the pressure balance from the SOLPS equations}

SOLPS-ITER solves the parallel momentum balance for each ion species. Summing up the individual momentum equations, momentum transfer between the ion species cancels out and the total momentum balance is obtained. Using the current conservation, the electric field contribution can be eliminated by introducing, amongst others, the static electron pressure. With $x$ the poloidal direction and $y$ the radial one, the following equation is derived (in steady state and in the absence of drift effects) $[45,50]$ :

$$
\begin{aligned}
\frac{b_{x}}{h_{x}} \frac{\partial}{\partial x}\left(p_{t o t}\right) & =-\nabla \cdot \Gamma_{m}^{v i s c}-\nabla \cdot \Gamma_{m}^{a n o m}+S^{m o m} \\
& -\frac{1}{h_{z} \sqrt{g}} \frac{\partial}{\partial x}\left(\frac{b_{x} h_{z} \sqrt{g}}{h_{x}}\right) \sum_{a} m_{a} n_{a}\left(V_{a}^{\|}\right)^{2} \\
& =S_{t o t}^{\text {mom }}
\end{aligned}
$$

with

$$
\begin{aligned}
p_{\text {tot }} & =\sum_{a} m_{a} n_{a}\left(V_{a}^{\|}\right)^{2}+\sum_{a} n_{a} T_{i}+n_{e} T_{e} \\
b_{x} & =\frac{B_{\text {poloidal }}}{B_{\text {tot }}} \\
\nabla \cdot \Gamma_{m}^{v i s c} & =\frac{1}{h_{z} \sqrt{g}} \frac{\partial}{\partial x}\left(\frac{h_{z} \sqrt{g}}{h_{x}} \sum_{a} \mu_{a x} \frac{\partial}{\partial x} V_{a}^{\|}\right) \\
& +\frac{1}{h_{z} \sqrt{g}} \frac{\partial}{\partial y}\left(\frac{h_{z} \sqrt{g}}{h_{y}} \sum_{a} \mu_{a y} \frac{\partial}{\partial y} V_{a}^{\|}\right)
\end{aligned}
$$

$$
\begin{aligned}
\nabla \cdot \Gamma_{m}^{a n o m} & =\frac{1}{h_{z} \sqrt{g}} \frac{\partial}{\partial x}\left(\frac{h_{z} \sqrt{g}}{h_{x}} \sum_{a} m_{a} V_{a}^{\|} \Gamma_{a x}^{a n o m}\right) \\
& +\frac{1}{h_{z} \sqrt{g}} \frac{\partial}{\partial y}\left(\frac{h_{z} \sqrt{g}}{h_{y}} \sum_{a} m_{a} V_{a}^{\|} \Gamma_{a y}^{a n o m}\right)
\end{aligned}
$$

and $h_{x}, h_{y}$ and $h_{z}=2 \pi R$ the metric coefficients, $\sqrt{g}=h_{x} h_{y} h_{z}$ the elementary cell volume, $\Gamma_{m}^{\text {visc }}$ the viscous momentum flux and $\Gamma_{m}^{a n o m}$ the momentum flux due to the anomalous particle transport. $S^{m o m}$ denotes the sources of momentum (see [45] for details), found to be dominated by momentum transfer to neutrals. The fourth term on the right hand side of the pressure balance is called the geometric term [50]. A part of the geometric term cancels out with the centrifugal force, which is part of $S^{m o m}$, but the other part has influence on the pressure balance and, hence, pressure balance is not expected even in attached conditions and in the absence of radial momentum transport. However, the geometric term is small if the total flux expansion is small $\left(R^{t} \sim R^{u}\right)$, which is the case here and pressure balance does approximately hold for attached conditions.

\section{References}

[1] M Wischmeier. High density operation for reactorrelevant power exhaust. Journal of Nuclear Materials, 463:22 - 29, 2015. plasma surface interactions 21.

[2] H. Zohm, C. Angioni, E. Fable, G. Federici, G. Gantenbein, T. Hartmann, K. Lackner, E. Poli, L. Porte, O. Sauter, G. Tardini, D. Ward, and M. Wischmeier. On the physics guidelines for a tokamak DEMO. Nuclear Fusion, 53(7):073019, jun 2013.

[3] RA Pitts, X Bonnin, F Escourbiac, H Frerichs, JP Gunn, T Hirai, AS Kukushkin, E Kaveeva, MA Miller, D Moulton, et al. Physics basis for the first ITER tungsten divertor. Nuclear Materials and Energy, page 100696, 2019.

[4] PC Stangeby. The plasma boundary of magnetic fusion devices. CRC Press, 2000.

[5] SI Krasheninnikov and AS Kukushkin. Physics of ultimate detachment of a tokamak divertor plasma. Journal of Plasma Physics, 83(5), 2017.

[6] AW Leonard. Plasma detachment in divertor tokamaks. Plasma Physics and Controlled Fusion, 60(4):044001, 2018.

[7] S Coda, M Agostini, R Albanese, S Alberti, E Alessi, S Allan, J Allcock, R Ambrosino, H Anand, $\mathrm{Y}$ Andrèbe, et al. Physics research on the TCV tokamak facility: from conventional to alternative scenarios and beyond. Nuclear Fusion, 59(11):112023, 2019.

[8] H Reimerdes, GP Canal, BP Duval, B Labit, T Lunt, WAJ Vijvers, S Coda, G De Temmerman, TW Morgan, F Nespoli, et al. Power distribution in the snowflake divertor in TCV. Plasma Physics and Controlled Fusion, 55(12):124027, 2013.

[9] J.R. Harrison, W.A.J. Vijvers, C. Theiler, B.P. Duval, S. Elmore, B. Labit, B. Lipschultz, S.H.M. van Limpt, S.W. Lisgo, C.K. Tsui, H. Reimerdes, 
U. Sheikh, K.H.A. Verhaegh, and M. Wischmeier. Detachment evolution on the TCV tokamak. Nuclear Materials and Energy, 12:1071 - 1076, 2017. Proceedings of the 22nd International Conference on Plasma Surface Interactions 2016, 22nd PSI.

[10] C Theiler, B Lipschultz, J Harrison, B Labit, H Reimerdes, C Tsui, WAJ Vijvers, JA Boedo, BP Duval, S Elmore, et al. Results from recent detachment experiments in alternative divertor configurations on TCV. Nuclear Fusion, 57(7):072008, 2017.

[11] O Février, C Theiler, J R Harrison, C Tsui, K Verhaegh, C Wüthrich, JA Boedo, H De Oliveira, BP Duval, B Labit, et al. Nitrogen-seeded divertor detachment in TCV L-mode plasmas. Plasma Physics and Controlled Fusion, 2020.

[12] K Verhaegh, B Lipschultz, B Duval, O Février, A Fil, C Theiler, M Wensing, C Bowman, DS Gahle, J Harrison, B Labit, C Marini, R Maurizio, H De Oliveira, H Reimerdes, U Sheikh, C Tsui, $\mathrm{N}$ Vianello, $\mathrm{W}$ Vijvers, and the EUROfusion MST1 team the TCV team. An improved understanding of the roles of atomic processes and power balance in divertor target ion current loss during detachment. Nuclear Fusion, 59(12):126038, 2019.

[13] A Fasoli, H Reimerdes, S Alberti, M Baquero-Ruiz, BP Duval, E Havlikova, A Karpushov, J-M Moret, M Toussaint, H Elaian, et al. TCV heating and divertor upgrades. Nuclear Fusion, 60(1):016019, 2019.

[14] JR Harrison, C Theiler, O Février, H de Oliviera, R Maurizio, K Verhaegh, A Perek, A Karpushov, B Lipschultz, BP Duval, et al. Progress toward divertor detachment on TCV within H-mode operating parameters. Plasma Physics and Controlled Fusion, 61(6):065024, 2019.

[15] A Fil, B Dudson, B Lipschultz, D Moulton, K Verhaegh, O Fevrier, M Wensing, EUROfusion MST1 teams, and TCV team. Identification of the primary processes that lead to the drop in divertor target ion current at detachment in TCV. Contributions to plasma physics, 58(6-8):746-750, 2018.

[16] M Wensing, BP Duval, O Février, A Fil, D Galassi, E Havlickova, Arthur Perek, H Reimerdes, C Theiler, K Verhaegh, et al. SOLPS-ITER simulations of the TCV divertor upgrade. Plasma Physics and Controlled Fusion, 61(8):085029, 2019.

[17] M Wischmeier. Simulating divertor detachment in the TCV and JET tokamaks. PhD thesis, École Polytechnique Fédérale de Lausanne, 2004.

[18] D Galassi, H Reimerdes, C Theiler, M Wensing, H Bufferand, G Ciraolo, P Innocente, Y Marandet, $\mathrm{P}$ Tamain, and the EUROfusion MST1 Team. Numerical investigation of optimal divertor gas baffle closure on tcv. Plasma Physics and Controlled Fusion, 2029

[19] I Yu Senichenkov, E G Kaveeva, E A Sytova, V A Rozhansky, S P Voskoboynikov, I Yu Veselova, D P Coster, X Bonnin, and F Reimold and. On mechanisms of impurity leakage and retention in the tokamak divertor. Plasma Physics and Controlled Fusion, 61(4):045013, mar 2019.

[20] V. Rozhansky, E. Kaveeva, I. Senichenkov, E. Sytova, I. Veselova, S. Voskoboynikov, and D. Coster. Electric fields and currents in the detached regime of a tokamak. Contributions to Plasma Physics, 58(6-8):540-546, 2018

[21] E Sytova, RA. Pitts, E Kaveeva, X Bonnin,
D Coster, V Rozhansky, I Senichenkov, I Veselova, $\mathrm{S}$ Voskoboynikov, and F Reimold. Comparing N versus $\mathrm{Ne}$ as divertor radiators in ASDEX-upgrade and ITER. Nuclear Materials and Energy, 19:72 78,2019

[22] S Wiesen, D Reiter, V Kotov, M Baelmans, W Dekeyser, AS Kukushkin, SW Lisgo, RA Pitts, V Rozhansky, G Saibene, et al. The new SOLPSITER code package. Journal of nuclear materials, 463:480-484, 2015.

[23] X Bonnin, W Dekeyser, $\mathrm{R}$ Pitts, D Coster, S Voskoboynikov, and S Wiesen. Presentation of the new SOLPS-ITER code package for tokamak plasma edge modelling. Plasma and Fusion Research, 11:1403102-1403102, 2016.

[24] S. Touchard, J. Mougenot, C. Rond, K. Hassouni, and X. Bonnin. AMMONX: A kinetic ammonia production scheme for EIRENE implementation. Nuclear Materials and Energy, 18:12 - 17, 2019.

[25] JW Davis and AA Haasz. Impurity release from low-Z materials under light particle bombardment. Journal of nuclear materials, 241:37-51, 1997.

[26] GM McCracken, RS Granetz, B Lipschultz, B Labombard, F Bombarda, JA Goetz, S Lisgo, D Jablonski, H Ohkawa, JE Rice, et al. Screening of recycling and non-recycling impurities in the alcator C-Mod tokamak. Journal of nuclear materials, 241:777$781,1997$.

[27] GP Maddison, C Giroud, GK McCormick, JA Alonso, B Alper, G Arnoux, PC da Silva Aresta Belo, MNA Beurskens, A Boboc, S Brezinsek, et al. Moderation of divertor heat loads by fuelling and impurity seeding in well-confined elmy h-mode plasmas on jet. Nuclear fusion, 51(4):042001, 2011.

[28] R Zagórski, G Telesca, G Arnoux, M Beurskens, W Fundamenski, K McCormick, JET-EFDA Contributors, et al. Self-consistent modeling of impurity seeded jet advanced tokamak scenarios. Journal of nuclear materials, 390:404-407, 2009.

[29] G Kawamura, H Tanaka, K Mukai, B Peterson, SY Dai, S Masuzaki, M Kobayashi, Y Suzuki, Y Feng, LHD Experiment Group, et al. Threedimensional impurity transport modeling of neonseeded and nitrogen-seeded LHD plasmas. Plasma Physics and Controlled Fusion, 60(8):084005, 2018.

[30] ME Fenstermacher, SL Allen, NH Brooks, DA Buchenauer, TN Carlstrom, JW Cuthbertson, EJ Doyle, TE Evans, P-M Garbet, RW Harvey, et al. The two-dimensional structure of radiative divertor plasmas in the diii-d tokamak. Physics of Plasmas, 4(5):1761-1773, 1997.

[31] O Février, C Theiler, H De Oliveira, B Labit, $\mathrm{N}$ Fedorczak, and A Baillod. Analysis of wallembedded langmuir probe signals in different conditions on the tokamak à configuration variable. Review of Scientific Instruments, 89(5):053502, 2018.

[32] K Verhaegh, B Lipschultz, BP Duval, JR Harrison, H Reimerdes, C Theiler, B Labit, R Maurizio, C Marini, F Nespoli, et al. Spectroscopic investigations of divertor detachment in TCV. Nuclear Materials and Energy, 12:1112-1117, 2017.

[33] K Verhaegh, Bruce Lipschultz, BP Duval, Alexandre Fil, M Wensing, Christopher Bowman, DS Gahle, et al. Novel inferences of ionisation and recombination for particle/power balance during detached discharges using deuterium balmer line spectroscopy. Plasma Physics and Controlled Fusion, 61(12):125018, 2019.

[34] SS Henderson, M Bernert, S Brezinsek, M Carr, 
M Cavedon, R Dux, B Lipschultz, MG O'Mullane, F Reimold, and ML Reinke. Determination of volumetric plasma parameters from spectroscopic NII and NIII line ratio measurements in the ASDEX upgrade divertor. Nuclear Fusion, 58(1):016047, dec 2017.

[35] T Ravensbergen, M van Berkel, SA Silburn, JR Harrison, A Perek, K Verhaegh, WAJ Vijvers, C Theiler, A Kirk, MR de Baar, et al. Development of a realtime algorithm for detection of the divertor detachment radiation front using multi-spectral imaging. Nuclear Fusion, 60(6):066017, 2020.

[36] MG Dunne, S Potzel, F Reimold, M Wischmeier, E Wolfrum, Lorenzo Frassinetti, M Beurskens, P Bilkova, M Cavedon, R Fischer, et al. The role of the density profile in the asdex-upgrade pedestal structure. Plasma Physics and Controlled Fusion, 59(1):014017, 2016.

[37] UA Sheikh, Mike Dunne, Lorenzo Frassinetti, Patrick Blanchard, BP Duval, Benoît Labit, Antoine Merle, Olivier Sauter, C Theiler, C Tsui, et al. Pedestal structure and energy confinement studies on TCV. Plasma Physics and Controlled Fusion, 61(1):014002, 2018

[38] Estera Stefanikova, Lorenzo Frassinetti, S Saarelma, A Loarte, I Nunes, L Garzotti, P Lomas, F Rimini, P Drewelow, U Kruezi, et al. Effect of the relative shift between the electron density and temperature pedestal position on the pedestal stability in jetilw and comparison with jet-c. Nuclear Fusion, 58(5):056010, 2018

[39] PC Stangeby and Chaofeng Sang. Strong correlation between D2 density and electron temperature at the target of divertors found in SOLPS analysis. Nuclear Fusion, 57(5):056007, 2017.

[40] B Lipschultz, JL Terry, C Boswell, JA Goetz, AE Hubbard, SI Krasheninnikov, B LaBombard, DA Pappas, CS Pitcher, F Wising, et al. The role of particle sinks and sources in Alcator C-Mod detached divertor discharges. Physics of Plasmas, 6(5):1907-1916, 1999.

[41] S.I. Krasheninnikov, A.Yu. Pigarov, and D.J. Sigmar. Plasma recombination and divertor detachment. Physics Letters A, 214(5):285 - 291, 1996.

[42] AS Kukushkin, SI Krasheninnikov, AA Pshenov, and D Reiter. Role of molecular effects in divertor plasma recombination. Nuclear Materials and Energy, 12:984-988, 2017.

[43] JA Goetz, B LaBombard, B Lipschultz, CS Pitcher, JL Terry, C Boswell, S Gangadhara, D Pappas, $\mathrm{J}$ Weaver, B Welch, et al. High confinement dissipative divertor operation on alcator C-Mod. Physics of Plasmas, 6(5):1899-1906, 1999.

[44] RA Pitts, BP Duval, A Loarte, J-M Moret, JA Boedo, D Coster, I Furno, J Horacek, AS Kukushkin, D Reiter, et al. Divertor geometry effects on detachment in TCV. Journal of Nuclear Materials, 290:940-946, 2001.

[45] R Schneider, X Bonnin, K Borrass, DP Coster, H Kastelewicz, D Reiter, VA Rozhansky, and BJ Braams. Plasma edge physics with B2-eirene. Contributions to Plasma Physics, 46(1-2):3-191, 2006.

[46] V Kotov and D Reiter. Two-point analysis of the numerical modelling of detached divertor plasmas. Plasma physics and controlled fusion, 51(11):115002, 2009 .

[47] D Moulton, J Harrison, B Lipschultz, and D Coster. Using SOLPS to confirm the importance of total flux expansion in Super-X divertors. Plasma
Physics and Controlled Fusion, 59(6):065011, 2017.

[48] B Lipschultz, B LaBombard, JL Terry, C Boswell, and IH Hutchinson. Divertor physics research on Alcator C-Mod. Fusion science and technology, 51(3):369-389, 2007.

[49] E. de la Cal, J. Guasp, A. Salas, D. Reiter, P. Börner, J.A. Alonso, R. Balbín, D. Carralero, C. Hidalgo, J.L. de Pablos, F.L. Tabarés, and D. Tafalla and. Spatially resolved H-emission simulation with EIRENE in TJ-II to study hydrogen atomic and molecular physics in low density, high temperature fusion edge plasmas. Nuclear Fusion, 48(9):095005, aug 2008.

[50] V Kotov, D Reiter, DP Coster, and AS Kukushkin. Verification of the 2D Tokamak edge modelling codes for conditions of detached divertor plasma. Contributions to Plasma Physics, 50(3-5):292-298, 2010. 